\title{
Erosion study of Fe-W binary mixed layer prepared as model system of RAFM steel
}

\author{
K. Sugiyama, J. Roth, V.Kh. Alimov ${ }^{\mathrm{a}}$, K. Schmid, M. Balden, S. Elgeti, F. Koch, \\ T. Höschen, M.J. Baldwin ${ }^{\mathrm{b}}$, R.P. Doerner ${ }^{\mathrm{b}}$, H. Maier, W. Jacob \\ Max-Planck-Institut für Plasmaphysik, Garching, Germany \\ ${ }^{a}$ Hydrogen Isotope Research Center, University of Toyama, Toyama, Japan \\ ${ }^{b}$ Center for Energy Research, University of California at San Diego, La Jolla, CA, USA
}

\begin{abstract}
Fe-W binary mixed layers were prepared as a model system for reduced-activation ferriticmartesitic (RAFM) steel for studying their dynamic erosion behaviour resulting from energetic deuterium (D) irradiation. This investigation aims towards an assessment of RAFM steels as plasmafacing material. The surface composition of the model layers is modified by D irradiation. W is enriched at the surface due to the preferential sputtering of Fe. The W surface concentration increases with $\mathrm{D}$ fluence. It depends on the D impinging energy as well as the initial $\mathrm{W}$ fraction of the Fe-W layer. No significant development of surface topography was observed within the examined conditions. The erosion yield of a Fe-W layer is comparable to that of pure Fe in the low-fluence range and decreases with increasing $\mathrm{D}$ fluence. These results indicate that the dynamic change of erosion yield is significantly correlated with the surface W enrichment. (144 words)
\end{abstract}

PSI-21 keywaords: Sputtering, Stainless steel, Surface analysis

PACS. No. 28.52.Fa (Fusion reactors. Materials)

52.40.Hf (Plasma-wall interactions; boundary layer effects; plasma sheaths)

81.65.Cf (Surface cleaning, etching, patterning)

52.77.Bn (Etching and cleaning)

*Corresponding author:

Kazuyoshi Sugiyama

Max-Planck-Institut für Plasmaphysik

Boltzmannstrasse 2, D-85748 Garching, Germany

TEL: +49-(0)89-3299-1492

FAX: +49-(0)89-3299-1212

E-mail: kazuyoshi.sugiyama@ipp.mpg.de 


\section{Introduction}

Reduced-activation ferritic martensitic (RAFM) steel, such as EUROFER [1], RUSFER [2] or F82H [3], is planned to be used as a structural material in a future fusion reactor [4-6]. A model in the European conceptual design studies for a future power plant has proposed the usage of bare RAFM steel tiles as a possible option for the recessed areas of plasma-facing components because of technological and economical advantages [7]. One main concern for the plasma-facing steel wall is the erosion rate which determines the lifetime of wall components. In recessed areas, steel surfaces face erosion mainly due to the bombardment of charge-exchange neutrals with a broad distribution of energies but strongly peaking at low energies. Along with the base material: iron (Fe), RAFM steels contain typical mid-Z steel elements (e.g. chromium, vanadium, manganese) as well as small amounts of high-Z elements such as tungsten (W) ( 0.45 at.\% for EUROFER and RUSFER, $\sim 1.0$ at.\% for $\mathrm{F} 82 \mathrm{H})$. Since the sputtering yield of the mid-Z elements including $\mathrm{Fe}$ is significantly higher than that of high-Z elements, the surface composition will change during erosion due to preferential sputtering of low-/mid-Z elements, resulting in an enrichment of high-Z elements at the surface. This change of surface composition directly affects the erosion of RAFM steels.

The erosion rate of steel walls must be numerically assessed for the exact tokemak plasma edge conditions by using dynamic sputtering calculation programs such as SDTrimSP [8], and thus reliable experimental data are necessary for the bechmarking of simulation code. In this sense, some exposures of RAFM steels to high-flux plasmas have been performed recently [9,10]. On the other hand, RAFM steel is a highly complicated composite material with crystal phases and grain structures, which is not an adequate system to check the validity of simulation codes. Therefore, in this study, Fe-W binary mixed layers are prepared as a model system to investigate the underlying elementary physical processes relevant in the sputtering of steel grades. The dynamic erosion behaviour resulting from energetic deuterium (D) irradiation is parametrically investigated under well-defined laboratory conditions.

\section{Experimental procedure}

\subsection{Layer preparation and pre-characterization}

The Fe-W layers were prepared by magnetron-sputter deposition in a device (Leybold, UNIVEX 450B) equipped with multiple targets using argon ( $\mathrm{Ar}$ ) as working gas. The compositioin of the Fe-W films can be varied by adjusting the imput power for each target independently. Lower-Z materials, such as graphite and silicon, were chosen as substrates to facilitate layer characterization. In this study, 
3 depositions were peformed with different deposition conditions. Substrates were not biased and not actively cooled during a deposition. The substrate was slightly heated by deposition particle bombardment, nevertheless, the temperature was generally below $370 \mathrm{~K}$. For each deposition, reference substrates (glass and graphite) were also put in, and used for the pre-characteriations. After each deposition, thickness of the deposited layer on the reference glass substrate was measured by a profilometer. Areal densities and composition of the layer were checked by Rutherford backscattering spectroscopy (RBS) using $3 \mathrm{MeV}^{4} \mathrm{He}^{+}$as the probe beam. The specifications of prepared layers are summarized in table 1 . Figure 1 shows RBS spectra of as-deposited layers obtained from each deposition batch. W concentrations determined by the RBS measurement were $0.5,0.7$ and 4.2 at.\%, respectively. The $\mathrm{W}$ concentrations of 0.5 and 0.7 at.\% are in the range of RAFM steels, and the higher $\mathrm{W}$ concentration of 4.2 at.\% was prepared for comparison to check the influence of the initial $\mathrm{W}$ fraction in the Fe-W layer on sputtering. The layer thickness was in the range of $250-290 \mathrm{~nm}$, which is optimized for the layer characterization, allowing clear separated Fe and W RBS spectra as shown in Figure 1. The pressure during a deposition was $0.3 \mathrm{~Pa}$ due to the Ar gas, while the background pressure is normally $10^{-6} \mathrm{~Pa}$. At this condition, oxygen $(\mathrm{O})$ concentration is typically in the range of $1 \sim 2$ at.\%. However the deposition batch \#1 (W: 0.5 at.\%) had relatively high $\mathrm{O}$ concentration of about 8 at.\% because of unexpected small leakage.

Table 1

Specification of the Fe-W model system layers prepared for this study.

\begin{tabular}{cccccc}
\hline Batch & W concentration & O concentration & Thickness & Substrate & Experiment \\
\hline$\# 1$ & 0.5 at.\% & $\sim 8$ at.\% & $260 \mathrm{~nm}$ & Graphite & HSQ, PISCES-A \\
$\# 2$ & 0.7 at.\% & $\sim 2$ at.\% & $250 \mathrm{~nm}$ & Silicon & HSQ \\
$\# 3$ & 4.2 at.\% & $\sim 1.5$ at.\% & $290 \mathrm{~nm}$ & Silicon & HSQ \\
\hline
\end{tabular}

\subsection{D irradiation and post-irradiation analyses}

Prepared layers were then irradiated by D ions in the "HSQ" setup [11] at IPP Garching which provides a mass-separated $\mathrm{D}$ ion beam at defined ion energy. As bombarding species $\mathrm{D}_{3}{ }^{+}$ions were used, correspondingly, the energy per deuteron is $1 / 3$ of the ion energy. The deuteron flux is typically $\sim 10^{19} \mathrm{Dm}^{-2} \mathrm{~s}^{-1}$. The used D energies were 100, 200, 500 and $1000 \mathrm{eV} / \mathrm{D}$. The irradiation fluence was varied in the range of $10^{21}-10^{24} \mathrm{Dm}^{-2}$. The sample was not actively cooled during irradiation, leading to a slight temperature rise to 310 up to $350 \mathrm{~K}$ depending on ion energy. Since the achievable fluence in HSQ is limited, some exposures were performed in the linear plasma device: PISCES-A [12] at 
University of California San Diego for higher fluences $\left(>1 \times 10^{24} \mathrm{Dm}^{-2}\right)$. The sample was also slightly heated by the plasma exposure up to $\sim 380 \mathrm{~K}$. The PISCES-A plasma consists of different D molecule ions calculated to be $\left(\mathrm{D}^{+}: \mathrm{D}_{2}{ }^{+}: \mathrm{D}_{3}{ }^{+}\right)=(30: 38: 32)$ [13]. In this study, only the $\mathrm{D}^{+}$fluence was considered for the sputtering evaluation, since the molecular ions result after break up of the molecules due to impact in $1 / 2$ and $1 / 3$ of the incident energy per deuteron and correspondingly, the contribution of those speices for the sputtering is expected to be very small. The sample was biased with $150 \mathrm{~V}$, resulting in $\mathrm{D}^{+}$incident energy of $140 \mathrm{eV}$ due to the target potential of $10 \mathrm{eV}$.

Change of areal densities of the layer after D irradiation / exposure was analyzed by RBS in the same way as for the pre-characterization. The erosion yield was then calculated by the change of areal density and the $\mathrm{D}$ fluence. The surface composition and elemental depth profile were analyzed by RBS and sputter X-ray photoelectron spectroscopy (XPS). Since numerical simulations have predicted that the surface composition change would be restricted to a very thin near surface layer of a few nano meters, the analysis with the best depth resolution possible was performed. Therefore, RBS measurements for the surface composition analysis were carried out in the Cornell geometry arrangement, i.e., using $1 \mathrm{MeV}^{4} \mathrm{He}^{+}$with an incident angle of $75^{\circ}$ to the surface normal. While the information depth for these settings is limited $(<30 \mathrm{~nm}$ ), an improved depth resolution of $\sim 3 \mathrm{~nm}$ is achieved. XPS was performed with a PHI 5600 ESCA system using an Al Ko source. For the depth profiling, $10 \mathrm{keV} \mathrm{Ar}^{+}$was used for the surface etching, and the the photoelectron spectra were periodically recorded. The surface morphology was examined by scanning electron microscopy (SEM), Helios NanoLab600, combined with an energy-dispersive X-ray (EDX) spectrometer (FEI and INKA, Oxford).

\section{Result and discussion}

\subsection{Surface composition change due to D irradiation}

Figure 2 shows SEM pictures obtained from Fe-W layer with the W concentration of 0.7 at.\% before and after $\mathrm{D}$ irradiation. The layer prepared by the magnetron-sputter deposition has initially a nano-scale structure, nevertheless, the EDX elemental mapping revealed that both Fe and $\mathrm{W}$ are homogeneously distributed over the layer surface, which is not the case with RAFM steel materials [9,10]. Even after $200 \mathrm{eV} / \mathrm{D}$ irradation to a fluence of $1.0 \times 10^{24} \mathrm{Dm}^{-2}$, the layer has no severe damage or cracks, no strong topography development, but the nano structure was slightly modified. The EDX mapping for the irradiated layer surface confirmed that distributions of $\mathrm{Fe}$ and $\mathrm{W}$ are still homogeneous even though the relative concentrations of Fe and $\mathrm{W}$ on the surface could be changed. Therefore, one can conclude that the irradiated surface was uniformly eroded under D irradiation 
conditions in this study, which ensures suitability of the layer as the model system to compare with numerical studies.

Figure 3 (a) shows the RBS spectra obtained from a Fe-W layer (W concentration of 0.7 at.\%) after D irradiations with $200 \mathrm{eV} / \mathrm{D}$ with different D fluences. The increase of the $\mathrm{W}$ surface concentration with increasing fluence is clearly visible by the increase of the $\mathrm{W}$ back scattering peak at about $900 \mathrm{keV}$. Simultaneously the edge of Fe signal (around $750 \mathrm{keV}$ ) changes, i.e., the relative surface Fe concentration decreases with fluence indicating that the $\mathrm{W}$ enrichment is due to the preferential sputtering of Fe. The $\mathrm{W}$ concentration in the surface peak for the highest fluence case $\left(1.0 \times 10^{24} \mathrm{Dm}^{-2}\right)$ is evaluated to be roughly 9 at.\%. The $\mathrm{W}$ enrichment varies depending also on the $\mathrm{D}$ impinging energy, as shown in figure 3 (b). For the same fluence $1.0 \times 10^{23} \mathrm{Dm}^{-2}$, the surface $\mathrm{W}$ concentration increases as ion energy from 100 to $500 \mathrm{eV} / \mathrm{D}$. However, the W profile after $1000 \mathrm{eV} / \mathrm{D}$ irradiation shows a slightly lower surface peak than at $500 \mathrm{eV} / \mathrm{D}$, but higher tail into the depth up to several tens nm. Note that XPS depth profiling (not shown here) gave qualitatively similar results to the RBS analysis.

Preliminary SDTrimSP calculations, however, predict that the highest $\mathrm{W}$ enrichment should appear in the lower energy range of $100-200 \mathrm{eV} / \mathrm{D}$, while the enrichment would be reduced in the higher energy range. This numerical prediction seems actually reasonable because the sputtering of $\mathrm{W}$ is expected to be negligibly small at energies below $200 \mathrm{eV} / \mathrm{D}$. Furthermore, the calculation shows much higher $\mathrm{W}$ surface concentrations than those found in the experiments. This discrepancy could be explained by both experimental and simulation uncertainties as follows. For the low energy D irradiation, the enrichment takes place in a very limited depth range which is likely shallower than the resolution of the applied depth profiling methods. In such a case, the apparent surface $\mathrm{W}$ concentration determined by the depth profiling becomes lower than the real $\mathrm{W}$ concentration at the top surface. Another posiblility could be the presence of a nano-scale surface roughness after D irradiation. Roughness generally broadens the measured $\mathrm{W}$ profile, resulting in an underestimation of the $\mathrm{W}$ concentration. The tail found in some RBS $\mathrm{W}$ profiles might also be due to a nano-scale roughness. On the other hand, the simulated Fe and $\mathrm{W}$ sputtering yields generally do not perfectly match the experimental one. The simulated Fe sputtering yield gives slightly higher values than the experimental data. And the simulated $\mathrm{W}$ sputtering yield tends to be underestimated near the threshold energy (around 200eV/D) and overestimated in the energy range of $>400 \mathrm{eV} / \mathrm{D}$ compared with the experimental data [11]. These could also contribute to the observed discrepancy.

\subsection{Sputtering yield of Fe-W binary layer}

The fluence dependence of the measured sputtering yields for the Fe-W layers is summarized in 
figure 4. Data for $\mathrm{D}$ energies above $500 \mathrm{eV}$ are, unfortunately, available only for $\mathrm{D}$ fluences up to $10^{23} \mathrm{Dm}^{-2}$ because the layer was not thick enough for higher fluences. One can generally assert that the sputtering yields of Fe-W layers seem rather constant with relatively high yield - comparable with or in some cases even slightly higher than that of $\mathrm{Fe}$ - in the lower fluence range of $<10^{22} \mathrm{D} \mathrm{m}^{-2}$, while a clear reduction can be observed at higher fluences. This is qualitatively consistent with the evolution of the $\mathrm{W}$ surface enrichment. PISCES-A data at $5 \times 10^{23} \mathrm{Dm}^{-2}$, where the fluence overlaps with the fluence range of HSQ experiments, shows reasonable fit to the HSQ data. Increase of the fluence up to $2 \times 10^{24} \mathrm{Dm}^{-2}$ results in further reduction of sputtering yield. Overall, the yields do not achieve an "equilibrium" state, but show continuous decrease within the examined fluence range. In the comparison between low (W: 0.5 and 0.7 at.\%) and high (W: 4.2 at.\%) W concentration layers, the high concentration layer shows faster and larger reduction of the yield. While the sputtering yield of low $\mathrm{W}$ concentration layer is reduced by a factor of 2 - 3 after low energetic D irradiation with $10^{24} \mathrm{Dm}^{-2}$, that of the high initial W concentration layer shows a reduction by a factor of 5 - 10 under same irradiation conditions. This supports the hypothesis that the $\mathrm{W}$ concentration determines the change of the sputtering yield of theses Fe-W layer. However, the reduction of the sputtering yield is substantially lower than the value expected from the measured $\mathrm{W}$ enrichment. A measured $\mathrm{W}$ surface concentration of $\sim 9$ at.\% after irradiation with $200 \mathrm{eV} / \mathrm{D}, 10^{24} \mathrm{Dm}^{-2}$ cannot explain a reduction of the yield by a factor of $\sim 2$. This could again be due to the underestimation of surface $\mathrm{W}$ concentration, as discussed above.

\section{Summary and outlook}

Fe-W binary mixed layers with different $\mathrm{W}$ concentrations were prepared as model system for studying sputtering of RAFM steel and the dynamic erosion behaviour resulting from energetic D ion bombardment. The surface composition was clearly changed for all irradiation conditions, i.e., $\mathrm{W}$ is enriched at the surface due to the preferential sputtering of Fe. The W surface concentration increases with $\mathrm{D}$ fluence and the enrichment depends on the D impinging energy as well as the initial $\mathrm{W}$ fraction in the Fe-W layer. No significant surface topography development was observed within the investigated parameter range, which confirms the suitability of the layer as the model system to compare with numerical studies. The sputtering yields of Fe-W layers decreased with increasing $\mathrm{D}$ fluence. The yields do not achieve an "equilibrium" state, but show continuous decrease within the examined fluence range. These results indicate that the dynamic change of the erosion yield strongly correlated with the $\mathrm{W}$ surface enrichment. However, as discussed above, there is quantitative discrepancy in the $\mathrm{W}$ enrichment between experimental observation and simulation, which is to be solved through further experimental and benchmarking efforts. 
As the future work, the sputtering behaviour of RAFM steel will be examined applying the same experimental processes as in this study to check the similarity / difference with the Fe-W model system layer.

\section{Acknowledgement}

The authors greatfully acknowledge J. Dorner, C. Falsini, M. Fußeder G. Matern and A. Weghorn for their technical assistance. This work was partially supported by EFDA-Power Plant Physics \& Technology task agreement under contract of WP12-PEX-03a-T03-01/IPP and has received funding from the Euratom research and training programme 2014-2018. 


\section{References}

[1] B. van der Schaaf, F. Tavassoli, C. Fazio et al., Fusion Eng. Des. 69 (2003) 197-203

[2] V.M. Chernov, M.V. Leonteva-Smirnova, M.M. Potapenko et al., Nucl. Fusion 47 (2007) 839

[3] S. Jitsukawa, M. Tamura, B. van der Schaaf et al., J. Nucl. Mater. 307-311 (2002) 179-186

[4] K. Ehrlich, Fusion Eng. Des. 56-57 (2001) 71-82

[5] R.L. Klueh, A.T. Nelson, J. Nucl. Mater. 371 (2007) 37-52

[6] N. Baluc, K. Abe, V.M. Chernov et al, Nucl. Fusion 47 (2007) S696-S717

[7] D. Maisinnier, I Cook, S. Pierre et al., Fusion Eng. Des., 75-79 (2005) 1173-1179

[8] W. Eckstein, R. Dohmen, A. Mutzke et al., “SDTrimSP”, IPP Report 12/3 (1997)

[9] V.Kh. Alimov, Y. Hatano, K. Sugiyama et al., Phys. Scr. T159 (2014) 014049

[10] J. Roth, K. Sugiyama, V. Kh. Alimov et al., submitted to J. Nucl. Mater.

[11] W. Eckstein, C. Garcia-Rosales, J. Roth et al., “Sputtering Data”, IPP Report 9/82 (1993)

[12] D.M. Goebel, G. Campbell, R.W. Conn, J. Nucl. Mater. 121 (1984) 277-282

[13] E.M. Hollmann and A. Yu. Pigarov, Phys. Plasmas, 9 (2002) 4330 


\section{Figure captions}

Figure 1

RBS (by $3 \mathrm{MeV}^{4} \mathrm{He}^{+}$with normal incidence to the target surface) spectra obtained from the prepared Fe-W layers (deposited on the reference graphite substrate). The width and intensity of each elemental signal correspond to the thickness and relative concentration, respectively.

Figure 2

SEM images obtaimed from the surface of Fe-W layer (W concentration: 0.7 at.\%) (a) as deposited and (b) after D irradiation with $200 \mathrm{eV} / \mathrm{D}, 1.0 \times 10^{24} \mathrm{D} \mathrm{m}^{-2}$.

Figure 3

RBS (by $1 \mathrm{MeV}^{4} \mathrm{He}^{+}$with incident angle of $75^{\circ}$ ) spectra obtained from $\mathrm{Fe}-\mathrm{W}$ layer (initial W concentration: 0.7 at.\%) after D irradiations with (a) $200 \mathrm{eV}$ to different fluences and (b) with different impinging energies to a fluence of $1.0 \times 10^{23} \mathrm{Dm}^{-2}$.

Figure 4

Fluence dependence of sputtering yields of Fe-W layers with (a) low W concentrations ( 0.5 and 0.7 at.\%) and (b) high W concentration (W concentration: 4.2 at.\%). 


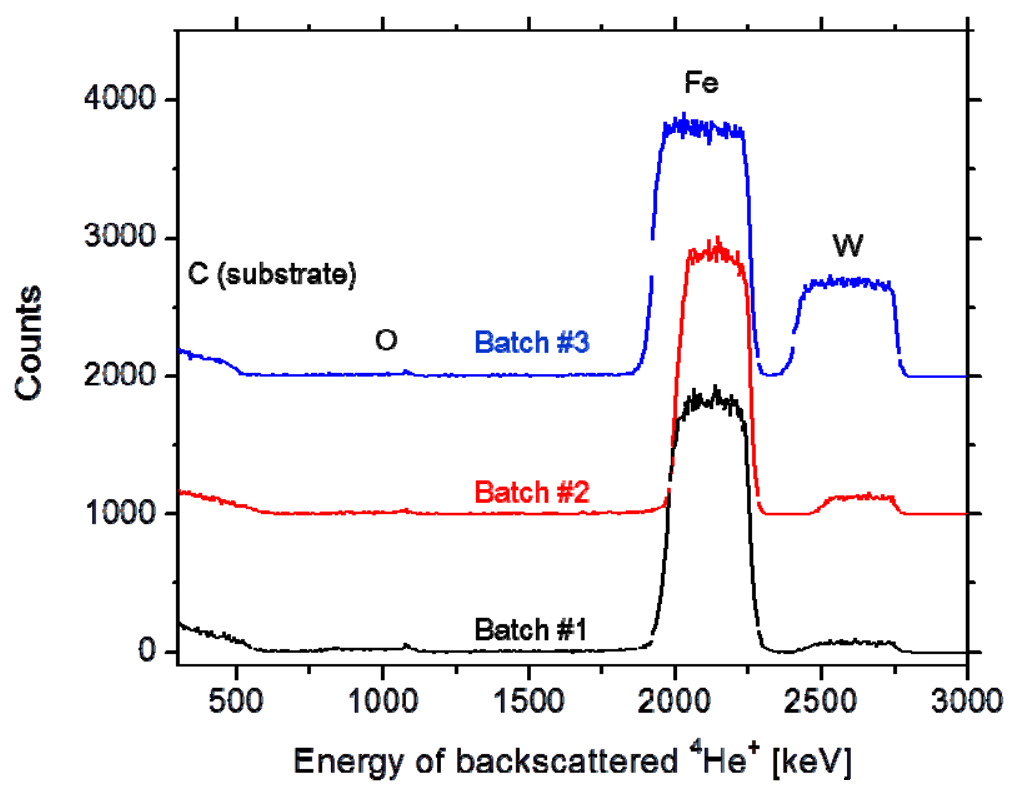

Figure 1

RBS (by $3 \mathrm{MeV}^{4} \mathrm{He}^{+}$with normal incidence to the target surface) spectra obtained from the prepared Fe-W layers (deposited on the reference graphite substrate). The width and intensity of each elemental signal correspond to the thickness and relative concentration, respectively. 

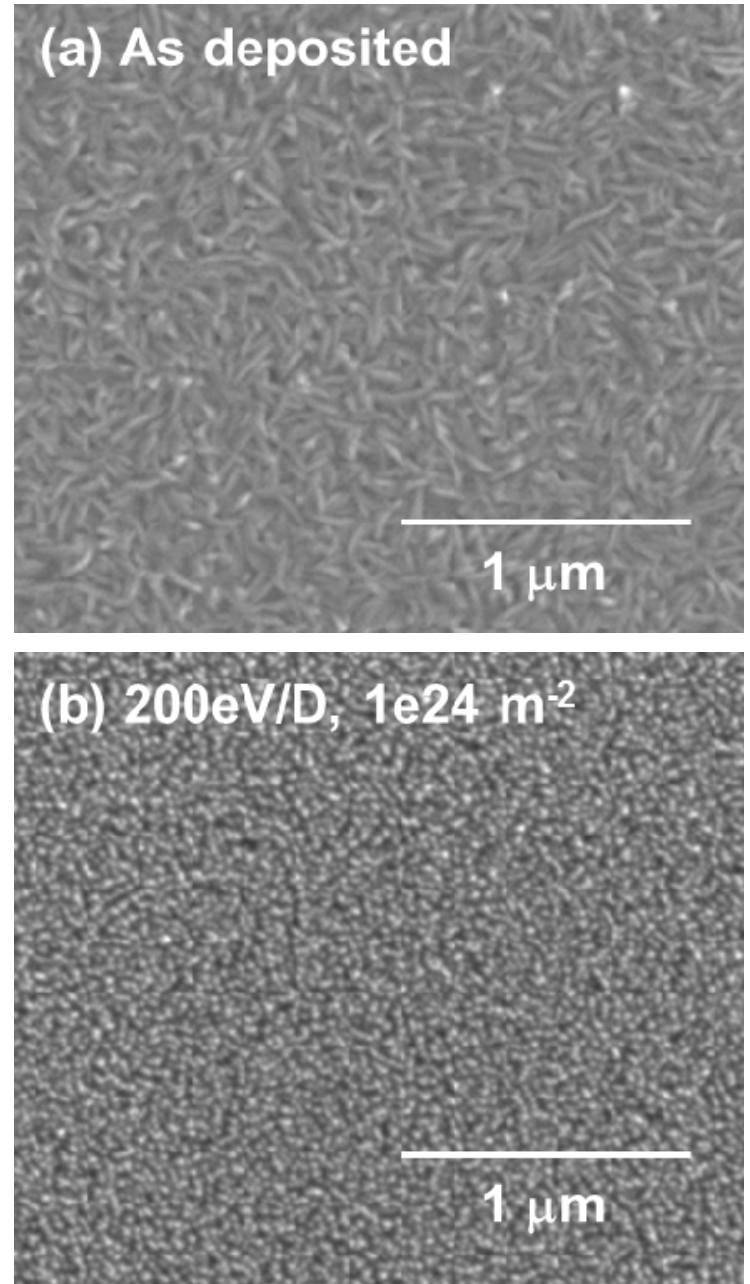

Figure 2

SEM images obtaimed from the surface of Fe-W layer (W concentration: 0.7 at.\%) (a) as deposited and (b) after D irradiation with $200 \mathrm{eV} / \mathrm{D}, 1.0 \times 10^{24} \mathrm{D} \mathrm{m}^{-2}$. (1 column) 

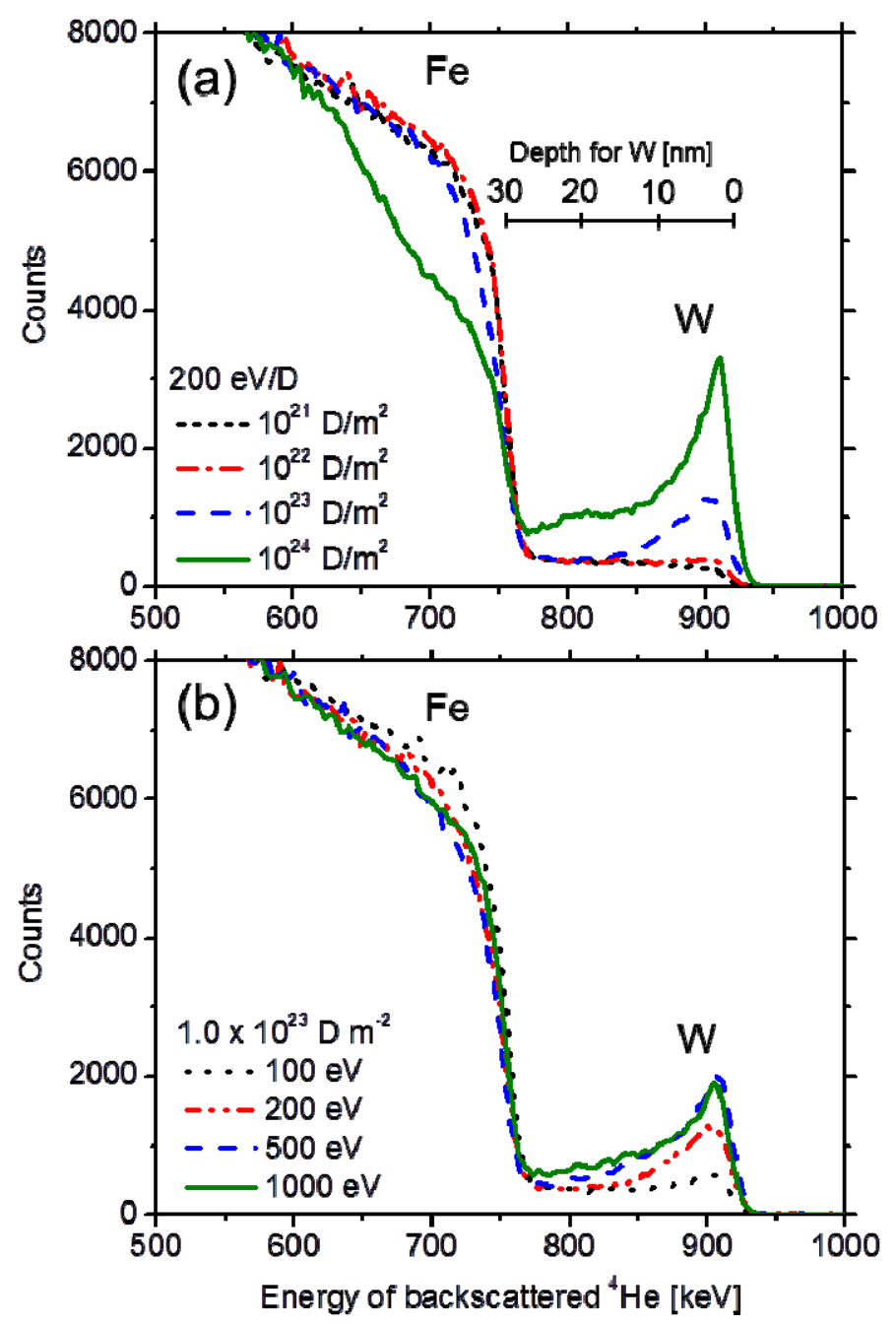

Figure 3

RBS (by $1 \mathrm{MeV} 4 \mathrm{He}^{+}$with incident angle of $7^{\circ}$ ) spectra obtained from $\mathrm{Fe}-\mathrm{W}$ layer (initial $\mathrm{W}$ concentration: 0.7 at.\%) after D irradiations with (a) $200 \mathrm{eV}$ to different fluences and (b) with different impinging energies to a fluence of $1.0 \times 10^{23} \mathrm{Dm}^{-2}$. (1 column) 

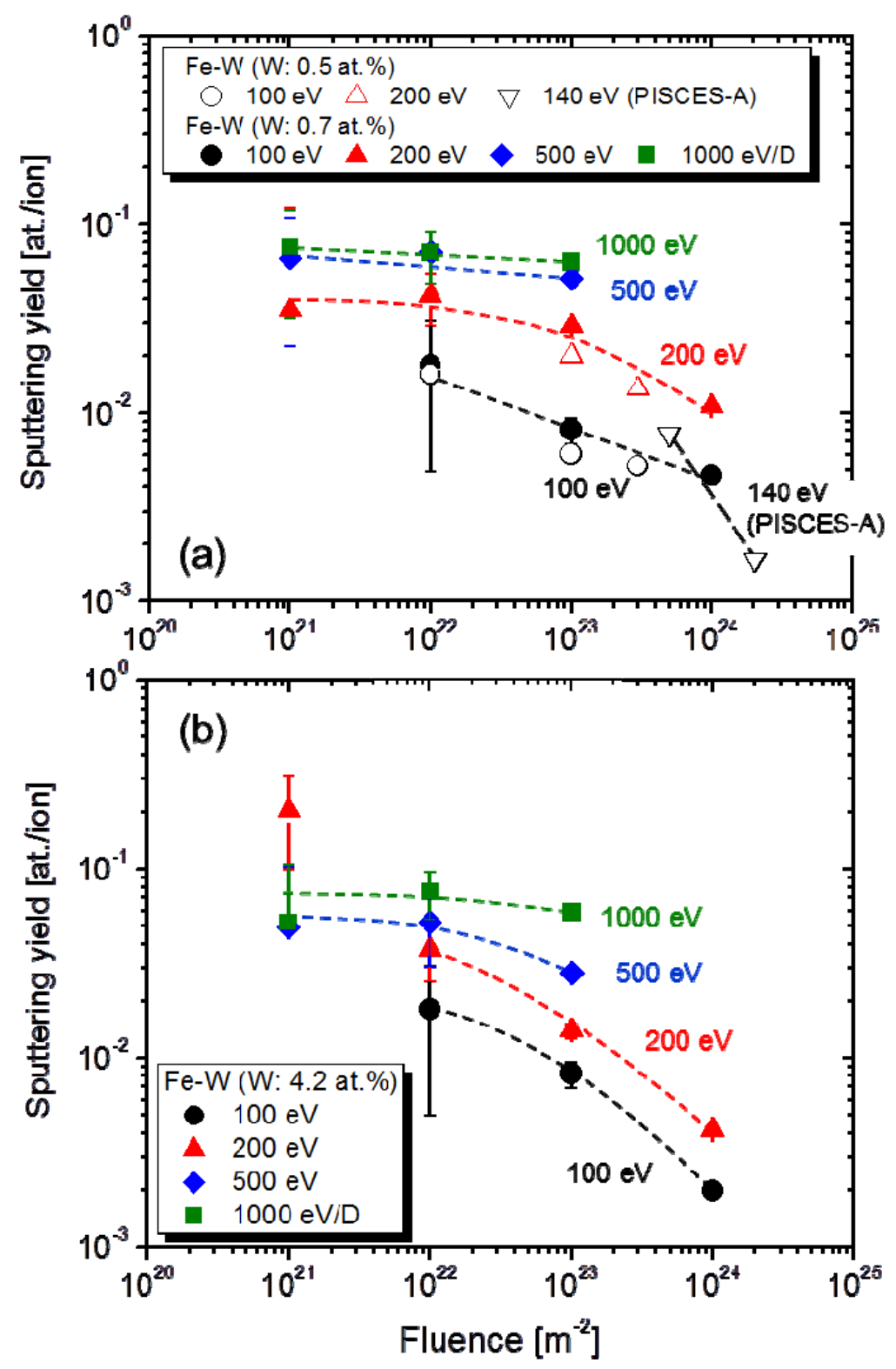

Figure 4

Fluence dependence of sputtering yields of Fe-W layers with (a) low W concentrations ( 0.5 and 0.7 at.\%) and (b) high W concentration (W concentration: 4.2 at.\%). (1 column) 